\title{
latrogenic calcinosis cutis in a child affected by Acute Lymphoblastic Leukemia
}

\author{
Teresa Perillo $^{1^{\star}}$, Giampaolo Arcamone ${ }^{1}$, Domenico Bonamonte ${ }^{2}$, Michele Pascone $^{3}$, \\ Nicola Santoro ${ }^{1}$
}

\footnotetext{
${ }^{1}$ Department of Pediatric Oncology, Policlinico Hospital, University of Studies, Bari, Italy;

*Corresponding Author: terryperillo@hotmail.com

${ }^{2}$ Department of Dermatology, Policlinico Hospital, Bari, Italy

${ }^{3}$ Department of Plastic Surgery, Policlinico Hospital, Bari, Italy
}

Received 29 November 2013; revised 25 December 2013; accepted 5 January 2014

Copyright (C) 2014 Teresa Perillo et al. This is an open access article distributed under the Creative Commons Attribution License, which permits unrestricted use, distribution, and reproduction in any medium, provided the original work is properly cited. In accordance of the Creative Commons Attribution License all Copyrights (C) 2014 are reserved for SCIRP and the owner of the intellectual property Teresa Perillo et al. All Copyright (C) 2014 are guarded by law and by SCIRP as a guardian.

\section{ABSTRACT}

latrogenic calcinosis cutis is a rare disorder that can be due to the intravenous administration of calcium or phosphate-containing infusions such as calcium gluconate or calcium chloride with extravasation. Fortunately, the course of calcinosis cutis is benign in immunocompetent children. The treatment remains supportive therapy. After about 6 months, there is no evidence of tissue calcification. We describe a 4-year-old girl with Acute Lymphoblastic Leukemia (ALL) who developed severe calcinosis cutis in the left humerus after extravasation of calcium gluconate during the treatment for the tumor-lysissyndrome-related hypocalcaemia. Surgical debridement and local wound care were not successful, and so a temporary suspension of chemotherapy was necessary to achieve complete healing of the lesion. Notwithstanding this complication, her ALL is in complete hematological remission after 8 months from the diagnosis. No functional or sensitive impairment due to the cutis has persisted.

\section{KEYWORDS}

\section{Calcinosis Cutis; Treatment; Immunocompromised; Leukemia}

\section{INTRODUCTION}

Calcinosis cutis is defined as an abnormal deposition of calcium phosphate in the skin and subcutaneous tissue. This uncommon disorder is classically divided into four subtypes: dystrophic, metastatic, idiopathic and iatrogenic [1]. The dystrophic variety occurs in damaged or traumatized tissues and the serum calcium/phosphorus ratio is found to be normal, whereas the metastatic variety is secondary to hypercalcemia or hyperphosphatemia. Idiopathic calcification occurs without any underlying metabolic disorder or tissue damage. Finally, iatrogenic calcinosis cutis is a rare variety that can be due to intravenous administration of calcium or phosphatecontaining infusions such as calcium gluconate or calcium chloride with extravasation [2]. Moreover, there are reports in the scientific literature of iatrogenic calcinosis following a single heel stick or applications of ncalcium chloride electrode paste in the neonatal period [3-7].

When the calcium extravasation is massive, leading to early signs of inflammation, the diagnosis of iatrogenic calcinosis cutis is easy and, on the clinical examination, lesions may be firm, diffusely erythematous, consisting of white to brownish papules or nodules or blister [2]. However, when the extravasation is small, intravenous infusion as the iatrogenic cause is difficult to corroborate as inflammatory signs may then be less marked and calcification delayed.

In any case, signs of inflammation usually appear several days after the administration of a calcium solution, and the mean period reported between the infusion and the appearance of a clinical lesion is 13 days (range, 2 hours to 24 days).

The initial radiographic finding following extravasation of calcium gluconate is soft-tissue swelling with no evidence of calcium density, whereas radiographs taken at 4 or 5 days to 3 weeks after infusion show a combination of patterns that may range from an amorphous mass localized at the site of injection to extensive areas of cal- 
cification, vascular or perivascular calcifications, and the rare annular variety [2].

There is no specific treatment available for this condition, although various treatments performed in single cases have been reported to be beneficial [8]. Fortunately, the course of calcinosis cutis is benign in immunocompetent children. The treatment remains supportive therapy. After about 6 months, there is no evidence of the tissue calcification. Areas of skin necrosis, if present, require debridement and local tissue care. Some of these cases might require subsequent skin grafting.

Here, we report a case of iatrogenic calcinosis cutis in an immunocompromised child affected by Acute Lymphoblastic Leukemia (ALL).

\section{CASE REPORT}

We describe a case of a 4-year-old girl affected by Bcell ALL. Once the diagnosis of ALL (Common-type) had been made, the young patient was started on intravenous infusion of prednisone according to the induction treatment of the AIEOP protocol 2009 for B lineage-ALL. On the third day of treatment with prednisone, despite having received the intravenous hyperhydratation, diuretic therapy (intravenous furosemide) and anti-uricolytic therapy (oral allopurinol) from the beginning, she developed a severe tumor lysis syndrome (TLS) with hyponatremia, hyperkaliemia, hypocalcemia, hyperuricemia, and acute renal failure with raised renal function parameters (blood urea nitrogen and creatinin). TLS-related hypocalcemia was treated by $10 \%$ calcium gluconate solution intravenous infusion at a dose of 3 $\mathrm{ml} / \mathrm{kg} /$ day for two days, then tapered to $1-2 \mathrm{ml} / \mathrm{kg} /$ day for 8 days and finally to $1 \mathrm{ml} / \mathrm{kg} /$ day for 15 days until normalization of serum calcium levels. On the third day of intravenous therapy with calcium gluconate, just after an episode of extravasation involving the venous access placed a few centimeters over the left elbow, the young patient complained of severe pain at the left humerus. A few hours later, a vast, erythematous area appeared, with an hyperchromic central zone, surrounded by yellow calcified papules. Soon after, the central area of the lesion necrotized, so that we could see a black slough in the middle of the wound. Doppler ultrasound of the zone involved excluded venous thromboses. The lesion was treated with daily disinfection and wound care with collagenase. One week later, the patient had completely recovered from renal failure, and the first doses of chemotherapy according to phase IA of induction were administered. During the first weeks of chemotherapy, there were no morphological changes in the lesion, and the cultures obtained from the eschar did not show any infection. 33 days after, at the end of phase Ia of induction with intravenous methylprednisolone $[50 \mathrm{mg} /$ $\mathrm{mq} /$ day for 20 days, tapered to $25 \mathrm{mg} / \mathrm{mq} /$ day for 3 days, and finally to $12.5 \mathrm{mg} / \mathrm{mq} /$ day for 3 days], intravenous Vincristine $[1.5 \mathrm{mg} / \mathrm{mq}]$ and Daunoblastin [30 $\mathrm{mg} / \mathrm{mq}]$-4 doses, intramuscular Peg-Asparaginase (1800 UI) - 2 doses, intrathecal methotrexate [12 mg] 3 doses, the girl underwent a "courettage" and a minor escharectomy surgical procedure. Histological analysis of the skin biopsy performed during the surgical procedure confirmed the clinical suspicion of "calcinosis cutis”. The day after (on day 40 after extravasation), phase Ib of induction chemotherapy was started, and a single dose of intravenous cyclophosphamide [1 g/mq], two cycles of four daily doses of intravenous arabinosyl cytosine $[75 \mathrm{mg} / \mathrm{mq} / \mathrm{day}]$ and daily oral 6-Mercaptopurinea were administered in two weeks. Despite the surgical procedure, the clinical appearance of the lesion deteriorated day by day, the ulcer extended and the nearby calcified areas enlarged (Figure 1).

A Magnetic Resonance Image (MRI) scan of the left arm showed a structural subversion of the adiposis, subcutaneous and fascial plane, a volumetric increase and an irregular structure of the muscular plane of the anterior compartment of the left arm, confirming that the soft tissues hardening was due to calcification deep into the muscular plane (Figure 2).

Blood samples were taken to check serum calcium and phosphorus levels, parathormone and calcitonin levels

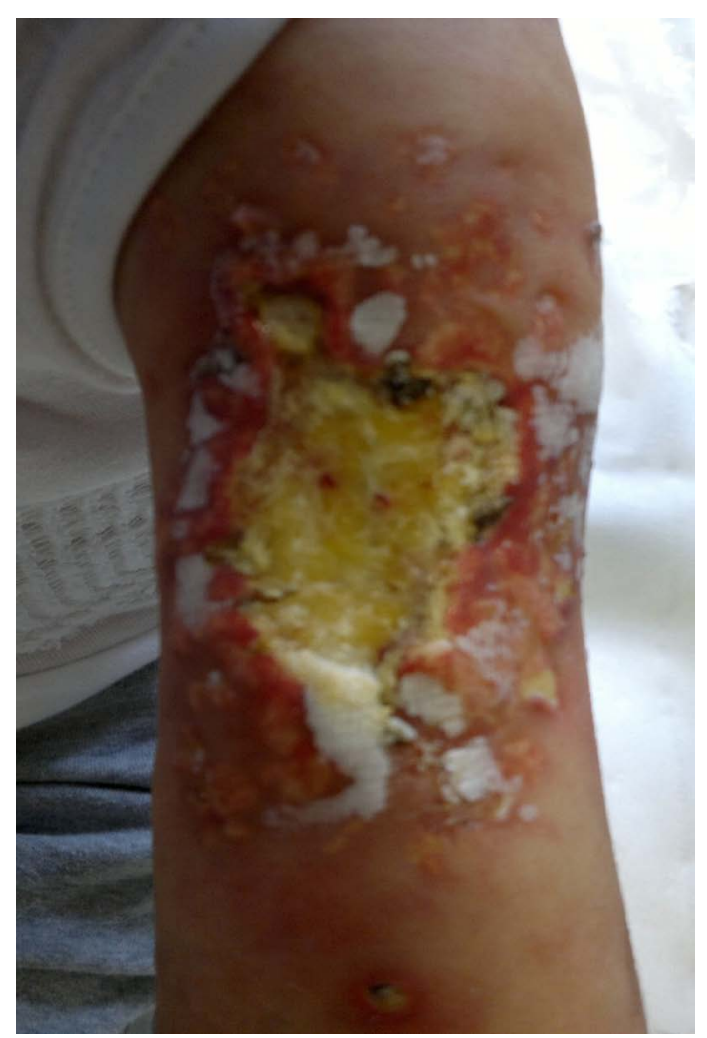

Figure 1. Deep ulcer surrounded by calcified papules. 


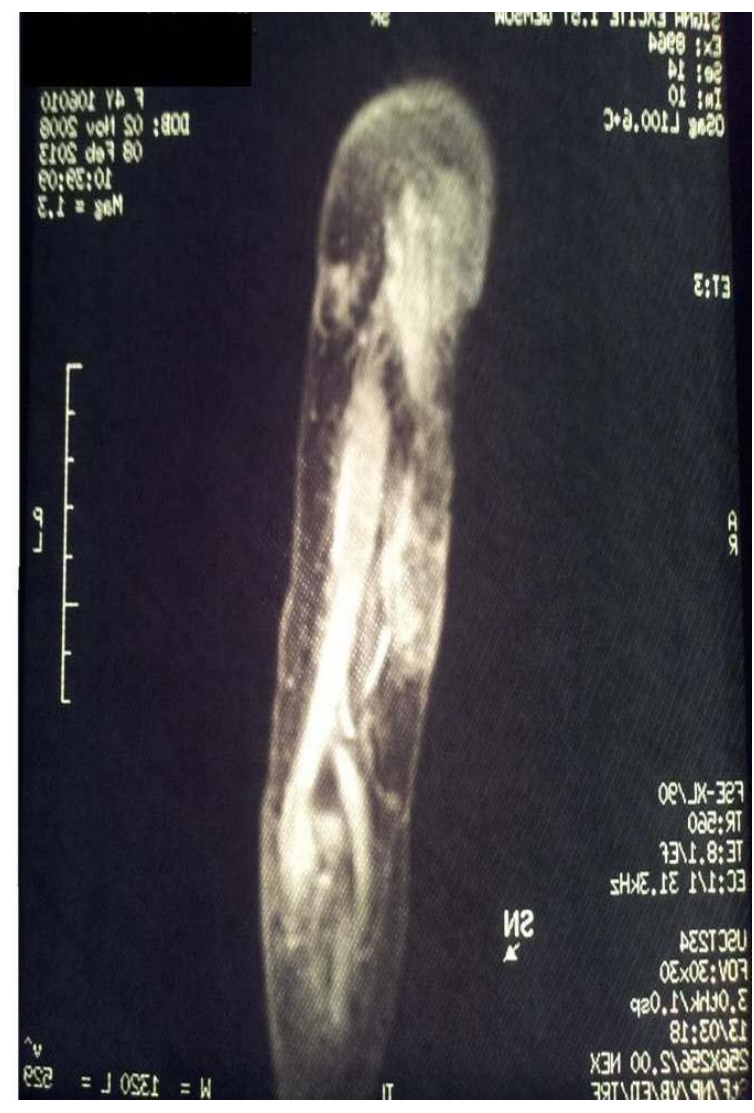

Figure 2. Magnetic resonance image scan of the left arm.

and urinary electrolytes in order to exclude the other causes of calcinosis cutis and confirm the extravasation following the intravenous administration of high-dose calcium gluconate as the cause of the lesion. Since no anomalies of the calcium/phosphorus metabolism were found, we concluded that the most probable explanation for the calcinosis cutis in our patient was a calcium-induced arterial spasm followed by vast extravasation of the high dose calcium gluconate solution. When the clinical conditions of the ulcer had worsened, we had decided to interrupt chemotherapy after the third cycle of arabinosyl cytosine (phase Ib of induction), and performed only clinical monitoring of the lesion during the following weeks. We decided not to perform a new surgical debridement with subsequent cutaneous autologous implantation as suggested by the plastic surgeon, because the new surgical procedure would require a continued interruption of the chemotherapy for at least 40 days, to allow tissues regeneration and the implanted skin flap to take root.

Moreover, as the young patient's haemathological parameters included leukopenia and thrombocytopenia during that period of time, the outcome of the implantation was uncertain.

Wound dressing was carried out at home, every two days, with collagenase and fitostimoline cream for the first two weeks after therapy suspension, and a silver cream thereafter. From day 82 after extravasation, together with a gradual return to normal values of the haemathological parameters, we witnessed a spontaneous development of granulation tissue from the bottom of the ulcer, and a softening of the surrounding tissues and margins. On day 78 from the beginning of chemotherapy, according to the AIEOP protocol, we performed a blood marrow aspirate, to evaluate the "minimal residual disease" by biomolecular studies. On the strength of the results of this test, the young patient was classified as a "low risk", despite having skipped a part of the chemotherapy. Therefore, we continued with Consolidation chemotherapy (four high-doses of intravenous methotrexate administered every fifteen days), and observed a gradual improvement of the lesion. The girl has now just completed the Reinduction phase of chemotherapy, the ulcer has healed with a massive reduction of soft-tissues calcifications (Figure 3).

Her ALL is in complete hematological remission after 8 months from diagnosis. The superficial cutaneous tissues over the lesion are dyschromic and will probably remain so, but this problem will be faced at the end of the chemotherapy.

\section{DISCUSSION}

The pathogenesis of calcinosis cutis complicating the intravenous calcium gluconate therapy can't be explained by the extravasation of calcium alone.

In fact, several authors have suggested that iatrogenic

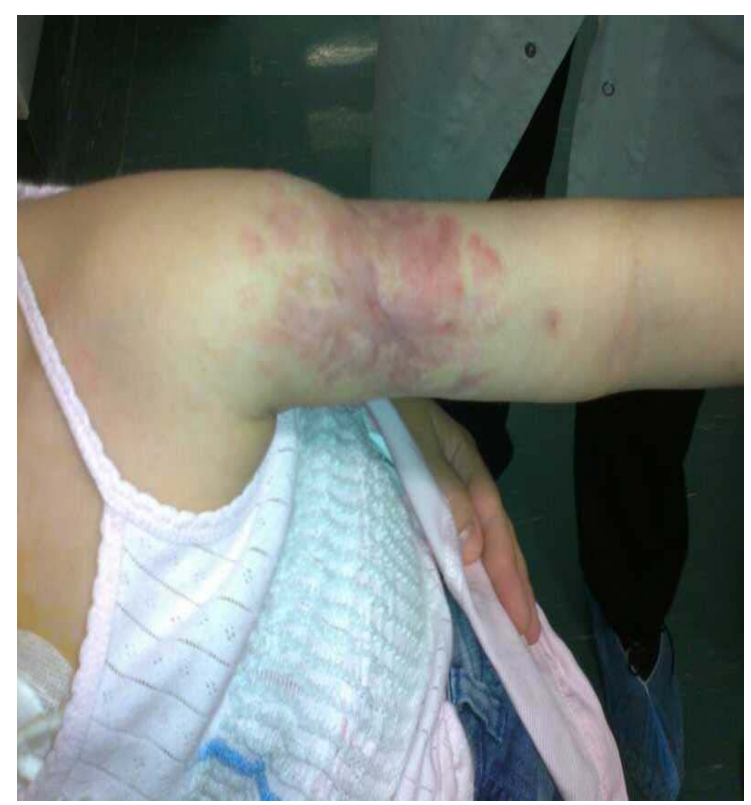

Figure 3. Healed ulcer with massive reduction of calcified areas. 
calcinosis following intravenous therapy can even occur without extravasation of calcium solution if the patient is receiving high doses of phosphorus or has hyperphosphatemia [9].

Therefore, the pathogenesis of this type of calcinosis cutis seems to be multifactorial, and theories to explain its pathogenesis have been recapitulated by RodriguezCano [2].

The various mechanisms that may act as triggering factors include temporarily elevated levels of serum and/or tissue calcium, local tissue damage resulting from phlebitis or repeated attempts to insert a peripheral line, and extravasation of a solution into the surrounding tissue. Local tissue damage increases cell membrane permeability, allowing a cytosolic influx of calcium phosphate, which precipitates in the cytoplasm. Extravasation of calcium solution, instead, causes collagen degeneration and soft-tissue necrosis.

Notably, Lestringant GG et al. have suggested that some alteration of the calcium metabolism at cellular and tissue level in leukemia may contribute to the pathogenesis of iatrogenic calcinosis cutis in patients affected by leukemia [10].

We therefore believe that our patient's lesion was caused by several mechanisms. We suppose that the high doses of calcium gluconate $(3 \mathrm{ml} / \mathrm{kg} /$ day $)$, may have induced an arterial spasm and a consequent vast extravasation of calcium gluconate solution. The local injury caused by the extravasated calcium, and the leukemia-induced alteration of the calcium metabolism at cellular and tissue level (as suggested by Lestringant GG), may have favored the release of further cellular calcium and the massive formation of calcium precipitates.

As stated above, calcinosis cutis is normally considered a self-limited condition that tends to resolve with conservative measures in 2 - 6 months [11].

However, in our patient, the skin calcinosis expanded despite normalization of the serum calcium level, the suspension of calcium supplementation and the surgical wound care. The deterioration of the lesion may be explained by the profound immunodeficiency induced by the induction phase of chemotherapy, which includes high doses of corticosteroids. In fact, any restitution ad integrum of damaged tissue is prevented by the effect of antiblastic drugs on actively regenerating tissues.

Colombatti et al. described a case of calcinosis cutis in a child affected by T-Acute Lymphoblastic Leukemia that developed during the induction treatment [12].

The extensive lesions caused by the extravasation of the calcium gluconate administered for a severe TLS, failed to improve the local wound care, surgical debridement and hyperbaric oxygen therapy. Three months after the extravasation, despite a substitution of phase Ib of induction by a less intensive chemotherapy, the mas- sive calcification extended. Successful intravenous treatment with Sodium Thiosulfate (STS) $(435 \mathrm{mg} / \mathrm{kg}$ three times a week) was carried out, and the eschars were almost completely healed after 6 months from extravasation. The authors concluded that STS could be considered a useful therapeutic option for iatrogenic calcinosis cutis in ALL patients.

Given the failure of local wound care and surgical debridement, we decided not to perform further therapeutic attempts because the efficacy of calcinosis treatments has been reported in single cases. Here is a notable lack of controlled clinical trials. No treatment is generally recognized as standard therapy, although various treatments have been reported to be beneficial, including warfarin, bisphosphonates, minocycline, ceftriaxone, diltiazem, alluminium hydroxide, probenecid, corticosteroids, intravenous immunoglobulin, carbon dioxide laser and extracorporeal shock wave lithotripsy [8].

Our decision has proved to be successful. The temporary suspension of chemotherapy, together with a gradual return to normal values of the haematological parameters, allowed a spontaneous improvement of the lesion. Moreover, the continued administration of antibiotics and careful disinfection of the ulcer guaranteed asepsis of the ulcer for three months, thus preventing any infection that would further complicate the patient's clinical conditions. Nevertheless, the temporary suspension of chemotherapy was not an easy decision to make, for fear of the possible negative consequences on the leukemic disease. Only after the excellent results of the minimal residual disease test, could we conclude that our choice was the correct one.

Our case highlights the fact that iatrogenic calcinosis cutis in immunocompromised patients is a real challenge for clinicians. Tailored treatments are necessary in patients undergoing chemotherapy because of the profound alterations of natural tissue regeneration mechanisms.

\section{ACKNOWLEDGEMENTS}

The authors wish to thank Mary V. C. Pragnell for the language revision and Christian Gerbino for the computer revision.

\section{REFERENCES}

[1] Angel, F.-F. (2011) Calcinosis cutis: Critical review. Acta Dermatovenerologica Croatica, 19, 43-50.

[2] Rodriguez-Cano, L., Garcia-Patos, V., Creus, M., Bastida, P., Ortega, J.J. and Castells, A. (1996) Childhood calcinosis cutis. Pediatric Dermatology, 13, 114-117. http://dx.doi.org/10.1111/j.1525-1470.1996.tb01415.x

[3] Sell, E.J., Hansen, R.C. and Struck-Pierce, S. (1980) Calcified nodules on the heel: A complication of neonatal intensive care. Journal of Pediatrics, 96, 473-475. http://dx.doi.org/10.1016/S0022-3476(80)80702-5 
[4] Leung, A. (1985) Calcification following heel sticks. Journal of Pediatrics, 106, 168. http://dx.doi.org/10.1016/S0022-3476(85)80493-5

[5] Cambiaghi, S., Restano, L. and Imondi, D. (1997) Calcified nodule of the heel. Pediatric Dermatology, 14, 494.

[6] Williamson, D. and Holt, P.J. (2001) Calcified cutaneous nodules on the heels of children: a complication of heel sticks as a neonate. Pediatric Dermatology, 18, 138-140. http://dx.doi.org/10.1046/j.1525-1470.2001.018002138.x

[7] Rho, N.K., Youn, S.J., Park, H.S., Kim, W.S. and Lee, E.S. (2003) Calcified nodule on the heel of a child following a single heel stick in the neonatal period. Clinical and Experimental Dermatology, 28, 502-503. http://dx.doi.org/10.1046/j.1365-2230.2003.01362.x

[8] Reiter, N., El-Shabrawi, L., Leinweber, B., Berghold, A. and Aberer, E. (2011) Calcinosis cutis. Treatment options. Journal of American Academy of Dermatology, 65, 15-22. http://dx.doi.org/10.1016/j.jaad.2010.08.039

[9] Lee, F.A. and Gwinn, J.L. (1975) Roentgen patterns of extravasation of calcium gluconate in the tissues of the neonate. Journal of Pediatrics, 86, 598-601. http://dx.doi.org/10.1016/S0022-3476(75)80161-2

[10] Lestringant, G.G., Masouyè, I., El-Hayek, M., Girardet, C., Revesz, T. and Frossard, P.M. (2000) Diffuse calcinosis cutis in a patient with congenital leukemia and leukemia cutis. Dermatology, 200, 147-150. http://dx.doi.org/10.1159/000018350

[11] Moss, J., Syrengelas, A., Antaya, R. and Lazova, R. (2006) Calcinosis cutis: A complication of intravenous administration of calcium gluconate. Journal of Cutaneous Pathology, 33, 60-62. http://dx.doi.org/10.1111/j.1600-0560.2006.00519.x

[12] Raffaella, C., Annapaola, C. and Tullio, I. (2009) Successful treatment of severe iatrogenic calcinosis cutis with intravenous sodium thiosulfate in a child affected by T-acute lymphoblastic leukemia. Pediatric Dermatology, 26, 311-315. http://dx.doi.org/10.1111/j.1525-1470.2008.00776.x 